\title{
ACERCAMIENTO A TRAVÉS DE UN ANÁLISIS ESPACIAL AL POBLAMIENTO DE LA EDAD DEL BRONCE FINAL Y LA EDAD DEL HIERRO EN EL SECTOR SUDOCCIDENTAL DE LA SUBMESETA NORTE
}

\author{
Rapprochment Through a Spatial Analysis to the Settlement of Late Bronze Age and \\ Iron Age in the Southwest of the Northern "Submeseta"
}

\author{
David HERNÁNDEZ SÁNCHEZ \\ Universidad de Salamanca \\ E-mail: davidhs66@hotmail.com
}

Fecha de recepción: 21-01-2010

Fecha de aceptación: 29-01-2010

\begin{abstract}
RESUMEN: El análisis espacial se ha confirmado en las últimas décadas como una metodología muy válida para avanzar en el conocimiento de las formas de poblamiento y la relación de los grupos humanos con el paisaje circundante. En este trabajo se muestran las bases de una investigación que aborda esta cuestión en los grupos humanos establecidos en el sector más sudoccidental de la Submeseta Norte durante las últimas etapas Protohistóricas. Se presenta un primer acercamiento que pretende confirmar la validez de la línea metodológica usada, ya que los datos de partida no permiten mayor profundidad por el momento. Se lleva a cabo la medición mediante un SIG de una serie de variables espaciales en su relación con los hábitats. Los resultados obtenidos son sometidos a análisis estadísticos multivariables, que nos llevan a la consecución de patrones de emplazamiento los cuales, puestos en relación con los datos cronológico-culturales conocidos, nos permiten vislumbrar marcas cronológico-culturales en el Paisaje.
\end{abstract}

Palabras clave: Edad del Bronce, Edad del Hierro, sudoccidente de Submeseta Norte, Poblamiento, Análisis espacial, SIG, análisis estadístico multivariable.

ABSTRACT: In the last few decades, spatial analysis has been confirmed to be a very valid methodology to advance towards the knowledge of the different forms of settlement and the relationship between groups of human beings and their environment. In the present 
work, it is presented the basis for a research which studies this issue in human groups settled in the southwest of the northern "Submeseta" during the last Protohistoric periods. It is shown as a first step that could confirm the validity of the methodology line used in this research, considering that the starting data doesn't allow further depth so far. The measuring is carried out with a GIS of a series of spatial variables in their relation with the habitats. The obtained results undergo multivariate statistical analysis from which we can infer different settlement patterns that, put in relation with the already known chronological-cultural data, allow us to discern chronological-cultural marks in the landscape.

Keywords: Bronze Age, Iron Age, Southwest of northing "Submeseta", Settlement, Spatial analysis, GIS, Multivariate statistical Analysis

\section{INTRODUCCIÓN}

En este trabajo se hace una breve recapitulación de los primeros resultados de una investigación en curso sobre el poblamiento de esta zona del occidente peninsular. Se pretende, a modo de inicio, comprobar la validez de la metodología propuesta, basada en un análisis espacial que toma los SIG y los análisis estadísticos multivariables como herramientas de trabajo, y el paisaje como parte básica del registro material. No obstante, el trabajo nos proporcionará algunas primeras conclusiones válidas sobre el poblamiento de la zona.

Bajo la premisa de la existencia de una lógica en el poblamiento prehistórico, unos intereses (económicos, estratégicos, simbólicos...) y una racionalidad a la hora de elegir la localización del grupo, el análisis sistemático e individual de los 69 yacimientos ${ }^{1}$ incluidos de acuerdo a una serie de variables espaciales nos permitirá conocer esta lógica a través de pautas regulares cuyas variaciones experimentadas en el tiempo o en relación con otras variables (cultura material, etc.) pueden ayudar a entender procesos de cambio y desarrollo del poblamiento experimentados por las sociedades prehistóricas de la zona. Se pasará de un análisis individual y objetivo de cada yacimiento a un tratamiento conjunto de los resultados que derivará en la obtención de patrones de comportamiento que, en último término, se pondrán en relación con datos cronológico-culturales para buscar posibles inferencias entre ellos.

La introducción de tiempos largos en el marco temporal, en el que se incluyen los periodos finales de la Edad del Bronce (Tardío y Final) y la Edad del Hierro hasta la romanización, se hace para comprobar con mayor nitidez las posibles marcas en el paisaje de cambios cronológico-culturales claros.

A pesar de la elección de unos límites evidentemente actuales², la provincia de Salamanca, esta zona cuenta con algunas fronteras naturales que, sin duda alguna, tendrán su repercusión en tiempos prehistóricos: al Noroeste 
las Arribes del Duero, que producen un corte difícilmente franqueable y al Sur las estribaciones montañosas del Sistema Central. En el marco escogido se engloban tres unidades morfoestructurales bien diferenciadas que condicionarán el poblamiento de la zona: las estribaciones montañosas al Sur, la llanura cerealista oriental y la penillanura occidental con un paisaje escarpado y encajado. El río Tormes actuará como eje vertebrador dividiendo llanura y

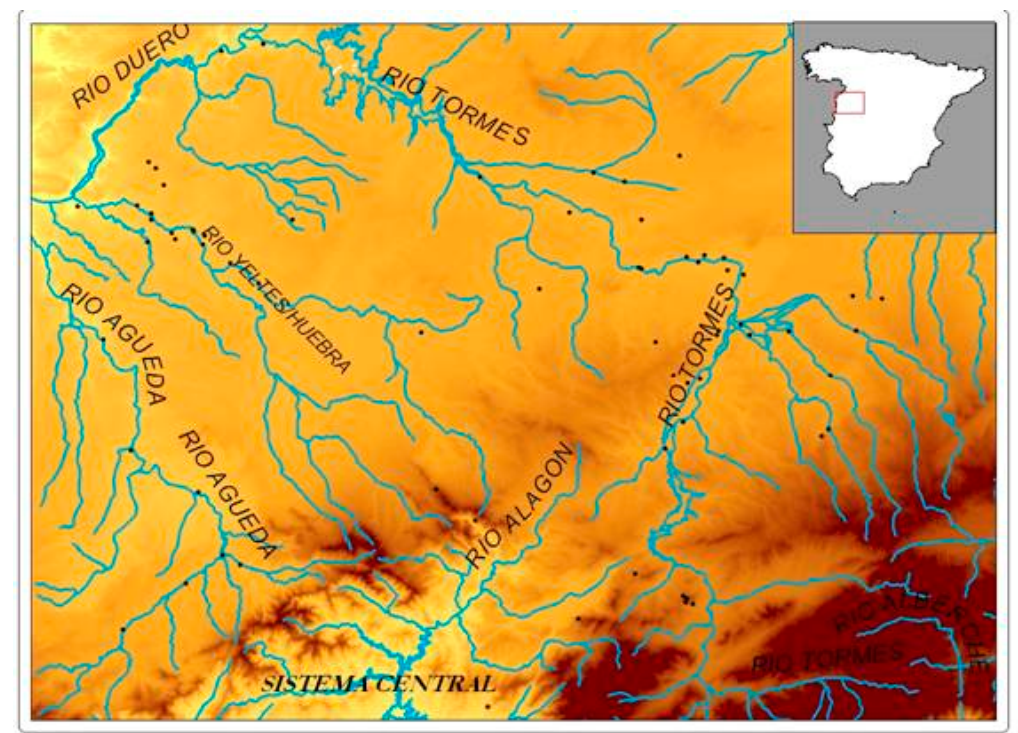

Fig. 1. Marco espacial. Los puntos representan los yacimientos incluidos en el análisis

penillanura por la que fluyen también varios cursos importantes, como son el Yeltes/Huebra, Águeda, Uces y el tramo más occidental del Tormes.

En esta primera fase de estudio no se han llevado a cabo trabajos de campo, utilizando como base una exhaustiva recopilación de los datos existentes. A pesar de una larga tradición investigadora en la zona³, son todavía muchas las lagunas y cuestiones por resolver: apenas tenemos secuencias estratigráficas que nos permitan asegurar la cronología de una gran parte de los yacimientos; del mismo modo, la inexistencia de excavaciones en extensión abundan en la escasez de conocimientos sobre hábitat, estructuras defensivas, adscripciones culturales, etc.; pobres son también los estudios polínicos, carpológicos, paleoclimáticos, etc.

En las últimas décadas se está trabajando en la superación de estas deficiencias desde diferentes frentes investigadores (Martín Valls, 1998; Fernández Posse, 1998; Álvarez Sanchís, 1999; López Jiménez, 2002), algunos de ellos con metodologías y posturas novedosas. Sin embargo, el estado de 
conocimientos previos en el que se enmarca este trabajo sigue siendo muy limitado, lo que sin duda afecta a su desarrollo y resultados.

\section{ANÁLISIS}

Las mediciones se realizan con una herramienta SIG, en concreto ArcView 3.3, en el que se han cargado las diferentes capas temáticas cartográficas, previamente filtradas y adaptadas, en la medida de lo posible, a la hipotética situación prehistórica. A partir de estas capas se medirá el comportamiento de los yacimientos respecto a cinco variables:

1/ La relación directa entre la altitud del punto escogido y los demás puntos de su entorno. La denominada altitud relativa. Su análisis permite comprobar si el yacimiento es dominante o no respecto a la mayor parte de los puntos de su entorno, y en qué grado. Se mide sobre el MDE de la zona y considerando tres rangos de distancias lineales: 1000, 2000 y 3000 m.

2/ La accesibilidad al curso de agua, midiendo la distancia en tiempo ${ }^{4}$ al punto más accesible de un curso de agua. Se parte de la idea de que el mantenimiento de una cabaña ganadera amplia exigiría un aprovisionamiento de agua en las inmediaciones superior al que permiten fuentes y manantiales, cuya presencia no se obvia, pero su situación en épocas pasadas escapa a nuestro conocimiento.

3 / El potencial productivo de los suelos respecto a tres entornos isócronos, de 0 a 30, de 30 a 60 y de 60 a 90 minutos. A partir del mapa de clases agrológicas actual, editando los factores posibles y teniendo siempre en cuenta que hablamos de una productividad teórica y no del uso real, se han creado tres categorías de suelos: intensivos (más propios para un uso agrícola), extensivos (más propios para uso silvopastoril) e improductivos.

4/ La accesibilidad a los recursos minerales. Para ello se ha editado el mapa metalogenético actual, filtrándolo a partir de los condicionamientos que supone el nivel tecnológico de la época, tanto para la extracción como para la reducción y manteniendo solamente los recursos teóricamente explotables por estos grupos. La simple edición del mapa permitirá cuestionar interpretaciones hechas sobre la influencia de estos recursos en el poblamiento de determinadas zonas.

5/ Por último, se mide la visibilidad desde el yacimiento respecto a tres entornos lineales de 800, 2000 y $5000 \mathrm{~m}$. Su medición con una herramienta SIG plantea aún muchos interrogantes e inconvenientes metodológicos. Éstos, unidos a otros problemas relacionados con la falta de datos sobre los yacimientos, no han permitido obtener resultados demasiado fiables, siendo parciales y desiguales. Su inclusión en el análisis estadístico conjunto, por 
tanto, se toma con mucha cautela.

De las mediciones se ha obtenido una tabla de resultados muy amplia y casi imposible de tratar de forma manual. Se someten a análisis estadísticos multivariables como método más apropiado para simplificarlos e interpretarlos, obteniendo toda la información posible de estos datos y su combinación.

La estadística es una herramienta ampliamente extendida en las investigaciones arqueológicas. Sin embargo, esta técnica concreta, la multivariable, tiene una implantación relativamente reciente en nuestro país (entre otros, Picazo Millán, 1991; Nocete Calvo, 1996). La posibilidad de operar con un número indeterminado de factores hace a esta técnica muy provechosa para su uso en Arqueología y especialmente en el estudio de patrones locacionales, como es el caso, donde convergen siempre multitud de factores, interrelacionados o no ${ }^{5}$.

En concreto se realiza un Análisis factorial de Componentes Principales (ACP) y un Análisis Cluster no-jerárquico de K-medias. El primero de ellos, el ACP, trabaja con un número indeterminado de realidades (en este caso, variables espaciales) observadas directamente sobre un conjunto de individuos (yacimientos), independientes unas de otras y todas con igual peso en el análisis. Opera reduciendo las variables originales y creando un grupo menor de nuevas variables, que son los factores o componentes, no observadas empíricamente y que explican la correlación o covarianza entre las originales. Básicamente, esta técnica intenta explicar la mayor proporción posible de correlación entre las variables originales eliminando así la información redundante o innecesaria incluida en las primeras. Cada factor o componente explica una realidad descrita por el grado de contribución de las diferentes variables originales en él. Posteriormente se obtienen grupos de yacimientos con similares comportamientos respecto a estos nuevos componentes.

En cuanto al Análisis Cluster, otorga flexibilidad en algunos aspectos en los que el ACP no la proporciona, por lo que matiza y completa sus resultados. La base de este tipo de análisis es más sencilla, ya que simplemente opera asignando casos (yacimientos) a un número preasignado de grupos (clusters o conglomerados) cuyas características no se conocen aún, pero que se basan en un conjunto de variables específicas. Proporciona, mediante el sistema de agrupación por acercamiento a una puntuación central, grupos de yacimientos previamente asignados con valores similares en las variables espaciales ${ }^{6}$.

Obtener la mejor rentabilidad de estos análisis ha conllevado la realización de múltiples ensayos, eliminando en el camino variables que no aportaban mucho, que podían introducir ruido o ser redundantes, hasta conseguir 
el modelo más completo y representativo de cada análisis. Estos modelos agrupan los yacimientos interpretando a partir de valores numéricos su comportamiento respecto a las variables espaciales estudiadas y permiten obtener de este modo las diferentes pautas en la elección del emplazamiento.

A partir de las agrupaciones resultantes del análisis estadístico, e incluyendo otros factores diferenciadores, como la presencia o ausencia de estructuras defensivas, así como una consideración más precisa de las características orográficas del emplazamiento (en alto, en llano, en ladera, etc.), se han elaborado los patrones de emplazamiento ${ }^{7}$, en concreto seis que engloban grupos amplios y un séptimo que agrupa yacimientos con comportamientos hoy por hoy singulares.

El primer patrón agrupa quince yacimientos en los que el interés a la hora de establecerse parece residir en la presencia en sus inmediaciones de suelos con potencial productividad intensiva. Los suelos más fértiles y húmedos, propios para una agricultura intensiva que no requiera excesiva inversión de trabajo y con pastos disponibles todo el año, aparecen en las vegas de los cursos de agua que fluyen por la llanura oriental, especialmente del Tormes. La mayoría de estos yacimientos parecen buscarlas intencionadamente para situarlas bien accesibles. Se trata de asentamientos situados en llano, ladera o suaves lomas, sin estructuras internas o de cerramiento conocidas, y donde la preeminencia y la visibilidad sobre el entorno quedan subordinados a los factores anteriormente mencionados.

Los cuatro asentamientos que componen el segundo patrón se emplazan en llano, ladera o pequeños berrocales. Las características son iguales a las del primer patrón, exceptuando el potencial productivo de los suelos del entorno que, en este caso, son mayoritariamente más extensivos, más duros para el cultivo y más propios para uso silvopastoril. Es un patrón poco consistente ya que incluye un escaso número de yacimientos de los que ni siquiera podemos asegurar su funcionalidad como hábitat, al conocerse únicamente escasos materiales en superficie.

También es reducido el número de asentamientos que componen el patrón tercero, seis en concreto. Se sitúan en alto, aprovechando cerros testigo de la llanura oriental. Parte de ellos contarán con estructuras defensivas y otra parte no, o no se conocen. En su entorno más accesible aúnan una importante proporción de suelos con productividad agrícola intensiva junto con un curso de agua cercano, como ocurría en el primer patrón. Pero en este caso, esto se conjuga con un claro interés por destacar sobre el entorno, aunque de forma no muy clara, ya que lo harán sólo sobre el más inmediato.

Con una mayor consistencia se establece el cuarto patrón. Un total de diecinueve yacimientos emplazados en lo alto de cerros o pequeñas mesas, en puntos poco accesibles y encajados en el paisaje que ofrece la Penillanura 
occidental y completados con estructuras defensivas. La preeminencia sobre el entorno no será un rasgo importante -existe sobre un entorno muy reducido y en la mayoría de los casos sin destacar demasiado-. Sí parece buscarse una relativa cercanía a un curso de agua, de importante caudal en casi todos los casos, que, por su encajamiento en el paisaje, refuerza la inaccesibilidad al emplazamiento. Los suelos del entorno, salvo alguna excepción, son mayoritariamente de productividad extensiva, propios para pastos, uso forestal y cultivos de ciclos largos, que necesitan de una agricultura más estable para posibilitar un mejor aprovechamiento de su fertilidad. Hay, además, una importante presencia de placeres estanníferos en los cursos que fluyen por las cercanías de la mayoría de estos asentamientos.

El quinto patrón define un grupo de quince yacimientos situados en alto, en zona montañosa o de penillanura, y donde el factor prioritario parece ser claramente la dominancia sobre todo el entorno, tanto el inmediato como el más alejado. No existen cursos de agua fácilmente accesibles y cuentan mayoritariamente con suelos de productividad extensiva, más propios para pastos y uso forestal, y donde mantener una buena productividad agrícola requiere de técnicas evolucionadas, ciclos de cultivo largos y, en algún caso de zona montañosa, trabajos de aterrazamiento. Sólo una parte de los yacimientos contarán con estructuras defensivas o de cerramiento.

Los siete yacimientos que componen el sexto patrón también se caracterizan por su emplazamiento en posiciones dominantes sobre el entorno, aprovechando cerros-testigo de la llanura oriental. No tienen estructuras defensivas o de cerramiento conocidas y cuentan con una importante proporción, en su entorno más accesible, de suelos con potencial productividad intensiva. En cuanto a la accesibilidad al curso de agua, no hay un comportamiento único, existiendo un grupo con buena accesibilidad y otro, donde ésta no es tan buena.

Por último, se han incluido en un séptimo patrón, que actúa de "cajón de sastre", cuatro asentamientos con comportamientos claramente singulares. En primer lugar, Cancho Enamorado, situado en lo alto de un cerro muy destacado sobre su entorno en las primeras estribaciones montañosas del Sureste. Sigue así un comportamiento de fuerte dominancia sobre todo el entorno, con altísimos valores de altitud relativa y visibilidad muy por encima de cualquier otro asentamiento analizado. Parece subordinar por completo a este hecho la productividad de los suelos del entorno -casi totalmente improductivos los más inmediatos- y la accesibilidad a un curso de agua -bastante alejados y de carácter menor-. Sin embargo, la presencia de estructuras domésticas parece confirmar la habitación del sitio (Maluquer, 1958).

Por otro lado, dentro de este último patrón están Mesa de Carpio Ber- 
nardo y Castillo del Carpio Bernardo, situados en lo alto de cerros testigo vecinos a la orilla del Tormes en su tramo más oriental, y sin estructuras de ningún tipo conocidas. Siguen un comportamiento en cierta manera similar al de Cancho Enamorado, mostrando también una clara intención de actuar como hitos en el paisaje. Pero en este caso, parecen prestar una mayor atención a la accesibilidad al curso de agua y a la presencia en su entorno accesible de suelos con cierta productividad.

Por último, el análisis ha singularizado el comportamiento de Castil de Cabras, asentamiento emplazado en alto en plena zona montañosa meridional y con estructuras de defensa o aterrazamiento visibles. Sigue un comportamiento en el que no hay dominancia ni visibilidad sobre el entorno, el tiempo de acceso al curso de agua más cercano es notable y los suelos casi totalmente improductivos en todo su entorno estudiado. Tampoco existen afloramientos minerales que puedan condicionar su presencia. Aquí, por tanto, ninguna de las variables analizadas parece determinar su emplazamiento, siendo necesario buscar otro tipo de motivaciones (estratégicas, relacionadas con rutas de paso, simbólicas, etc.) para su explicación.

INTERPRETACIÓN DE LOS RESULTADOS. HACIA UN MODELO DE POBLAMIENTO PARA LA ZONA

Los yacimientos que se han incluido en el estudio cuentan con una funcionalidad clara o presumible como establecimientos de población ${ }^{8}$ con una atribución segura en unos casos y dudosa en otros, al segmento temporal investigado. Este marco temporal tiene en el periodo broncíneo una clara adscripción en la zona más oriental a la cultura Cogotas I Pleno (XIII-XI a. C.) y Avanzado (XI-IX a. C.), con yacimientos de atribución segura, como La Aceña o Teso del Cuerno ${ }^{9}$ para el primero y Castillo y Teso del Carpio Bernardo o Cancho Enamorado para el periodo avanzado. Aún así, la consistencia de estas fechas y la adscripción cultural de algunos yacimientos se ponen actualmente en tela de juicio. Son importantes las dudas que se mantienen también sobre la situación de la zona más occidental de penillanura en estas últimas etapas broncíneas, donde son escasas las muestras de hábitat y la adscripción cultural de estos pocos restos está aún en el aire (Santonja, 1998)

Mayores problemas conllevan los siglos posteriores de transición entre la Edad del Bronce y la Edad del Hierro, los siglos IX-VIII a. C., con un registro hasta el momento muy limitado y sin teorías claras sobre la adscripción cultural de la zona (Fabián García, 1999; López Jiménez, 2002). En cuanto a la Edad del Hierro, los datos son mayores, principalmente en el Hierro II (V-III a. C.), periodo al que se adscriben los castros occidentales más cono- 
cidos (Yecla la Vieja, Las Merchanas o El Castillo de Saldeana, por ejemplo) y otros yacimientos con registros bastante bien conocidos (Teso de las Catedrales o Cuesta de Santa Ana). Sin embargo, la falta de excavaciones sistemáticas nos hace tener un conocimiento precario sobre la estructuración interna, el origen de los sistemas defensivos o la propia cronología exacta y adscripción cultural de la mayoría de estos yacimientos. La etapa inmediatamente anterior, el Hierro I, plantea mayores dudas, principalmente por la falta de registros que no permite conocer con claridad la extensión de la cultura Soto de Medinilla, presente claramente en estaciones occidentales como Cerro de San Vicente y Ledesma, y apenas tener datos sobre la zona más occidental.

Este breve repaso nos indica como apenas unos pocos sitios cuentan con una adscripción cronológica y/o funcional clara, moviéndose la mayoría en posiciones dudosas.

Como parte final del trabajo, los patrones obtenidos se ponen en relación con los datos cronológico-culturales conocidos de antemano, buscando de este modo posibles inferencias entre ellos que indiquen marcas en la relación de los grupos humanos con el paisaje y que se puedan poner en común con cambios cronológico-culturales o de otro tipo. El paisaje actuaría así como un valor material más del registro arqueológico (Parcero Oubiña, 2002; Orejas Saco del Valle, 1998).

La presentación de los datos cronológico-culturales en la parte final del trabajo ha sido intencionada, queriendo mostrar con ello que no han intervenido en la elaboración de los patrones. Se analiza el espacio físico para acercarse al tiempo, usando el primero para intentar definir el segundo y no determinando el primero a partir del segundo. Soy consciente, no obstante, de las limitaciones de este ensayo interpretativo, determinado, además de por las limitaciones metodológicas, por lo exiguo de los datos de partida. No obstante, resulta posible obtener algunas conclusiones y vislumbrar un posible modelo de poblamiento para la zona:

Valorando los patrones de una forma general -es decir, sin detenerse en las singularidades existentes- y considerando el poblamiento desde una perspectiva de larga duración, se advierten en ellos cambios significativos que guardan relación con las dos grandes etapas marcadas por la cultura material (Edad del Bronce y Edad del Hierro). Básicamente, nos hablan de una evolución hacia el sedentarismo y una mayor representatividad del yacimiento en el paisaje.

Los patrones que ofrecen una atribución más segura y que pondrían de manifiesto esta evolución serían el primer patrón, aglutinante de la mayoría de los yacimientos con adscripción a Cogotas I Pleno, establecidos en llano en las vegas fértiles de los ríos, especialmente en el tramo más oriental del 
Tormes, y sin apenas dejar marcas en el paisaje; y el cuarto patrón, que engloba los castros más occidentales del Yeltes/Huebra-Águeda, paradigmáticos del Hierro II en la zona, caracterizados por una clara sedentarización y bien marcados $^{10}$ en un paisaje que ofrece peores condiciones productivas a cambio de una mejor situación estratégico-defensiva (cerca de grandes ríos que podrían actuar como rutas de paso y aprovechando el medio natural escarpado para crear defensas).

Entrando ya en un terreno más hipotético, se puede seguir afinando en la interpretación de los patrones. A raíz de los datos cronológico-culturales con los que contamos, podemos relacionar con la Edad del Bronce, además del mencionado Patrón 1, casos singulares del séptimo patrón. Un Cogotas I Pleno de características ya mencionadas en el Patrón 1 y un último momento ya avanzado de la cultura (Patrón $7^{11}$ ) con muestras de un cambio de modelo en el que prima mucho la dominancia sobre el entorno y con indicios que indican una mayor permanencia en el sitio, cuestiones que podrían ponerse en relación con el control de rutas de paso $^{12}$. El particular caso de Cancho Enamorado, incluido en este último patrón, explicaría un fuerte factor estratégico o simbólico en la elección del emplazamiento, por las limitadas condiciones productivas del lugar escogido.

Del mismo modo, podemos corresponder con la Edad del Hierro los patrones 3, 4, 5 y 6, los cuales incluyen, como característica común, asentamientos ya sedentarizados y marcados en el paisaje, aunque manteniendo comportamientos diferentes en su relación con éste en cuanto a dominancia sobre el entorno y productividad del medio circundante. Así, distinguimos un grupo de asentamientos en la zona de las Arribes y otro en la zona serrana (Patrón 5), el mencionado grupo del Yeltes/Huebra-Águeda (Patrón 4) y un grupo de asentamientos en la llanura oriental (patrones 3 y 6). Estos diferentes modelos podrían corresponderse con adecuaciones de una misma cultura a zonas ecológicas diferentes (las diferentes unidades morfoestructurales que componen la zona) o a grupos culturales y/o cronológicamente distintos. El carácter de este trabajo y los datos existentes no permiten profundizar en estos aspectos.

Creo necesario pararme a comentar los resultados del cuarto patrón, por englobar los asentamientos del Yeltes/Huebra-Águeda, considerados tradicionalmente como los característicos de la Edad del Hierro en la zona y, como tales, los que mayor peso en las investigaciones han tenido. En cuanto a ellos, en primer lugar cabe matizar la creencia tradicional que los define como puntos dominantes en el entorno. A la vista de los resultados esta interpretación quedaría invalidada, definiéndose más bien como puntos mimetizados o casi escondidos en el paisaje.

Asimismo, la creencia de la riqueza de recursos minerales como causa 
de la anormal concentración de castros en esta zona (Martín Valls, 1998:166; Álvarez Sanchís, 1999:92-93) ha quedado también matizada a partir de los resultados obtenidos en la variable de recursos minerales. La pertenencia de todos ellos a un mismo patrón otorga datos a favor de su consideración como entidad arqueológica, pero a raíz de la edición del mapa de recursos minerales se comprueba que el presumido potencial minero de la zona no es tal ${ }^{13}$, por lo que es necesario buscar nuevas interpretaciones a este poblamiento, como ya va ocurriendo (López Jiménez, 2002).

El análisis espacial, por último, nos ha permitido también contrastar otras hipótesis planteadas por la cultura material, como es la particularidad del conjunto del Cerro del Berrueco o la pertenencia de Castil de Cabras a una entidad arqueológica distinta a la de sus vecinos serranos y propia de culturas más meridionales (Esparza y Blanco, 2008:85-87). Ha permitido también obtener datos a favor de la inclusión de yacimientos con atribución no segura en una determinada cultura o cronología, como es el caso de Castro de la Septa, cuya cronología, insinuada por su inclusión en un patrón claro de la Edad del Bronce (Patrón 1) parece corresponderse con los resultados de recientes excavaciones.

A pesar de lo limitado del ensayo, se han obtenido los patrones, se han conseguido algunas interpretaciones temporales de ellos y se han vislumbrado rasgos de un posible modelo de poblamiento para la zona, con la consecuente validación por ello de la metodología y de las herramientas usadas. Parece, por tanto, que esta línea de investigación, de la que este trabajo apenas marca un inicio, puede resultar fructífera. Se debe continuar por este camino con análisis espaciales más precisos, variados y completos, y, sobre todo, con la ampliación de datos básicos sobre estos grupos (cronológicos, materiales, culturales, etc.) para relacionar con los especiales, que sólo nos puede otorgar la realización de trabajos de prospección y excavación, los cuales se deben afrontar inexcusablemente para poder seguir avanzando.

\section{BIBLIOGRAFÍA}

Álvarez Sanchís, J. (1999): Los Vettones. Madrid: Real Academia de la Historia.

Esparza Arroyo, A.; Blanco González, A. (2008): "El solar de Vettonia antes de los vettones". En M. Almagro Gorbea (Ed.), Arqueología vettona: la meseta occidental en la Edad del Hierro. Alcalá de Henares: Museo Arqueológico Regional: 81-93.

Fabián García, F. J. (1999): "La transición del Bronce Final al Hierro en el Sur de la Meseta Norte. Nuevos datos para la sistematización”. Trabajos 
de Prehistoria, 56 (2):161-180.

Fernández Posse, M.D. (1998): La investigación protohistórica en la Meseta y Galicia. Madrid: Síntesis.

Gómez Moreno, M. (1967): Catálogo Monumental de España. Provincia de Salamanca. Madrid: Dirección General de Bellas Artes.

López Jiménez, O. (2002): Protohistoria del Occidente de la Meseta Norte. Tesis Doctoral inédita.

Maluquer de Motes, N. (1956): Carta Arqueológica de España. Salamanca. Salamanca: Diputación Provincial de Salamanca. Servicio de investigaciones arqueológicas.

- (1958): Excavaciones arqueológicas en el Cerro del Berrueco. Salamanca: Universidad de Salamanca.

Martín Valls, R. (1998): “La Edad del Hierro”. En J.L. Martín (Ed.), Historia de Salamanca (Vol. Prehistoria y Edad Antigua. Salamanca: Centro de Estudios Salmantinos: 137-163.

Morán Bardón, C. (1946): Reseña bistórico-artística de la provincia de Salamanca. Salamanca: Universidad de Salamanca.

Nocete Calvo, F. (1996): "Un modelo de aplicación de análisis multivariante a la prospección arqueológica: la definición de la unidad geomorfológico donde se establece el asentamiento". Arqueología espacial, 15: 7-35.

Orejas Saco del Valle, A. (1998): "El estudio del Paisaje: visiones desde la Arqueología". Arqueología espacial, 19-20: 9-19.

Parcero Oubiña, C. (2002): La construcción del Paisaje Social en la Edad del Hierro en el Noroeste Ibérico. Ortigueira: Fundación Fisterra.

Picazo Millán, J.V. (1991): “Contribución del análisis estadístico para la diferenciación de grupos culturales durante la Edad del Bronce en el Sur del Sistema Ibérico (Teruel, España)". Archeología e calcolatori, 2: 79-108.

Santonja, M. (1998): “Los tiempos prehistóricos". En J.L. Martín (Ed.), Historia de Salamanca (Vol. Prehistoria y Edad Antigua. Salamanca, Centro de Estudios Salmantinos: 19-122.

\section{Notas}

\footnotetext{
${ }^{1}$ Todos ellos, con mayor o menor seguridad dentro del rango temporal establecido en el trabajo y con una función clara o presumible como hábitat. Los datos con los que se cuentan en el momento de esta investigación no permiten una mayor precisión en este inventario inicial. No obstante, el análisis se realiza de una forma objetiva e individual a cada uno de
} 
los yacimientos, por lo que no existen apriorismos crono-culturales ni de otro tipo que condicionen los resultados.

${ }^{2}$ Se tuvieron en cuenta aspectos más actuales, como los inconvenientes a la hora de incluir en la investigación territorios pertenecientes a diferentes zonas administrativas (provincias, comunidades, países).

${ }^{3}$ Gómez Moreno hará una amplio inventariado patrimonial a principios del siglo XX (Gómez Moreno, 1967), seguido por los trabajos del Padre Morán (entre otros, Morán,1940) y con un avance científico definitivo en los trabajos de Maluquer de Motes, destacando su Carta arqueológica (Maluquer, 1956)

${ }^{4}$ Para calcular estas mediciones en tiempo se han creado los polígonos isócronos de cada yacimiento

${ }^{5}$ Por otra parte, es importante no olvidar que en los estudios espaciales la información que se maneja es casi siempre sesgada, lo que limita los resultados del análisis.

${ }^{6}$ Por ejemplo, un grupo de yacimientos con altitud relativa en 1000 m muy alta, mala accesibilidad al agua y gran proporción de suelos de potencial productividad intensiva en 90 minutos

${ }^{7}$ Su número es menor que el de los grupos resultantes del análisis estadístico, ya que para su elaboración se han tenido en cuenta las características en el comportamiento con un carácter más estructural. Los análisis estadísticos diferencian valores, y con los patrones buscamos diferenciar comportamientos, por lo que algunas agrupaciones separadas por el análisis estadístico se han agrupado en un mismo patrón al indicar sus valores un comportamiento similar.

${ }^{8}$ Algunos claros y otros más dudosos, en todo caso, yacimientos cuya función no es claramente diferente a la de hábitat, como pueden ser dólmenes, etc.

${ }^{9}$ Habría que envejecer el origen de este último hasta el Bronce Medio/Protocogotas, pero parece bastante claro que durante la etapa Cogotas I Pleno se mantendrá habitado

${ }^{10}$ Las estructuras defensivas y su situación en lugares topográficamente representativos lo indican. Con ello no se quiere decir que sean dominantes en el paisaje, que no lo son. Se trata de conceptos diferentes.

${ }^{11}$ En concreto Mesa del Carpio Bernardo, Castillo del Carpio Bernardo y Cancho Enamorado. Castil de Cabras parece responder a otros condicionantes.

${ }^{12}$ En este momento comienza el auge del comercio con el suroeste peninsular, en el que jugará un papel fundamental como ruta de paso la posteriormente conocida como Ruta de la Plata. Estos yacimientos su situarán en sus inmediaciones.

${ }^{13}$ Los afloramientos ferruginosos explotables desaparecen al filtrar el mapa, quedando únicamente un importante número de placeres estanníferos en los ríos que fluyen por esta zona. 


\begin{tabular}{|l|l|}
\hline Yacimiento & Cronologia/cuitura \\
\hline El Palomar & Cogotas I \\
El Soto & Cogotas I dudoso \\
Las Cabrillas & Cogotas I \\
Los Homos & Bronce Medio-Final dudoso \\
Bercimuelle & Cogotas I \\
Las Ollas & Cogotas I \\
La Acera & Cogotas I \\
Tierras de Garn & Cogotas I \\
Raya Espino/Torreperales & Cogotas I \\
El Regado & Cogotas I \\
Las Barceras & Cogotas I \\
Teso de la Hojita & Cogotas I dudoso \\
Los Pinares & Cogotas I \\
El Torrejón & Entre Bronce Final y Hierro I \\
Castro de la Senta & Entre Bronce Medio y Hierro I \\
& \\
\hline
\end{tabular}

Fig 2. Tabla con los yacimientos incluidos en el patrón.

\begin{tabular}{|l|l|}
\hline Yacimiento & Cronologia / cuitura \\
\hline Los Quemados & Edad del Bronce impreciso \\
Cortinas del Rio & Hierro I-Hierro II \\
Las Paredejas & Hierro I-Hierro II \\
Los Temizos & Hierro I dudoso \\
\hline
\end{tabular}

Fig 4. Tabla con los yacimientos incluidos en el patrón.

\begin{tabular}{|l|l|}
\hline Yacimiento & Cronologia /cultura \\
\hline Peña Blanca & Cogetas I-Cogetas I Avanzado \\
Teso del Cuemo & Cogotas I \\
El Bejarano & Cogotas I \\
Ciudad Rodrigo & Hierro II \\
Cerro de San Vicente & Hierro I-Hierro II \\
Teso de las Catedrales & Hierro II \\
\hline
\end{tabular}

Fig 6. Tabla con los yacimientos incluidos en el patrón.

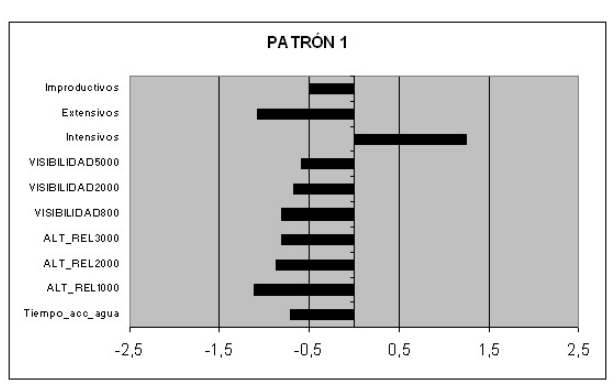

Fig 3. Media de los resultados estadísticos de los yacimientos del patrón. Los valores son relativos.

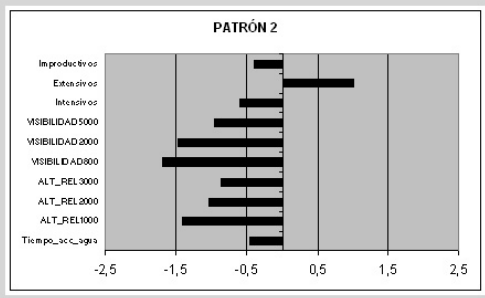

Fig 5. Media de los resultados estadísticos de los yacimientos del patrón. Los valores son relativos.

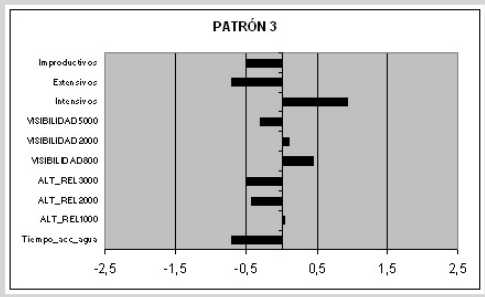

Fig 7. Media de los resultados estadísticos de los yacimientos del patrón. Los valores son relativos. 


\begin{tabular}{|c|c|}
\hline Yacimiento & Cronologia/cultura \\
\hline Huertamontes & \begin{tabular}{|l|} 
Bronce Final dudoso \\
\end{tabular} \\
\hline Picón del Rey & Bronce Final dudoso \\
\hline Los Ocuestos. & Hierro II \\
\hline Iruenta & Hiero II dudoso \\
\hline La Plaza & Hieno II dudoso \\
\hline Los Castillos de Gema & Hiero dudoso \\
\hline La Cabeza de Béiar. & Hierro dudoso \\
\hline Las Merchanas & Hieno II \\
\hline El Castillo de Saldeana & Hieno II \\
\hline El Guijo/El Castillo & Hierro dudoso \\
\hline Casa de Quiquín & Hieno dudoso \\
\hline Castillo de Saldañuela & Hierro II dudoso \\
\hline Herguijuela de Ciudad Rodrigo & Hieno I \\
\hline Picón de la Mora & Hiero I-Hierro II \\
\hline Ledesma-Plaza de San Martín & Hieno I-Hieno II \\
\hline Los Castillo de Pozos de Hinojo & Hiero dudoso \\
\hline Yecla la Vieja & Hierro II \\
\hline Castelmag & Hieno II dudoso \\
\hline & Hierro dudoso \\
\hline
\end{tabular}

Fig 8. Tabla con los yacimientos incluidos en el patrón.

\begin{tabular}{|l|l|}
\hline Yacimiento & Cronologia / cultuła \\
\hline Peñas de la Noria & Edad del Bronce dudoso \\
Cortinas de la Iglesia & Bronce Final dudoso \\
Las Cañadas & Cogotas I \\
Cabezo Castillo & Hiero dudoso \\
La Dehesa I & Hiero II \\
Los Tejares & Hierro II \\
Cabeza de Montako & Hierro II dudoso \\
La Corona & Hiero II \\
Cerro de San Pelayo & Hierro I-Hiero II \\
La Corvera & Hierro dudoso \\
Valdelascarnanas/Tejoneras & Hiero II \\
Virgen del Castillo & Hierro I-Hierro II \\
Cerro de San Cristóbal & Hierro II dudoso \\
Lerilla & Hierro I dudoso \\
\hline
\end{tabular}

Fig 10. Tabla con los yacimientos incluidos en el patrón.

\begin{tabular}{|l|l|}
\hline Yacimiento & Cronologia/cuitura \\
\hline Tordillos & Cogotas I dudoso \\
Teso de Valdecidiel & Cogetas I dudoso \\
Curbbre de la Flecha & Cogotas I \\
Castañeda/Matacán & Cogetas I \\
Camino de Cantaracillo & Bronce Final-Hierro I dudoso \\
Cuesta de Santa Ana & Hiero II \\
Teso de Utrera & Hierro II dudoso \\
\hline
\end{tabular}

Fig 12. Tabla con los yacimientos incluidos en el patrón.

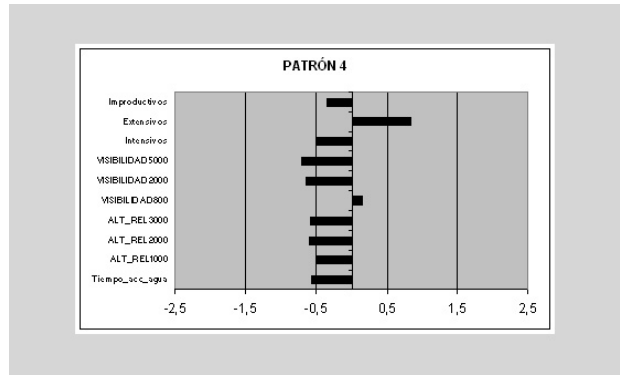

Fig 9. Media de los resultados estadísticos de los yacimientos del patrón. Los valores son relativos.

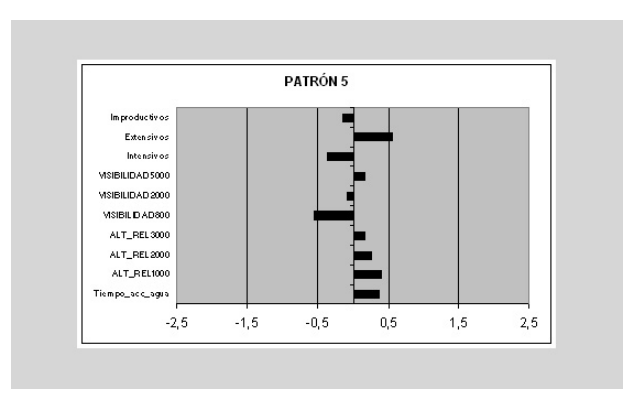

Fig 11. Media de los resultados estadísticos de los yacimientos del patrón. Los valores son relativos.

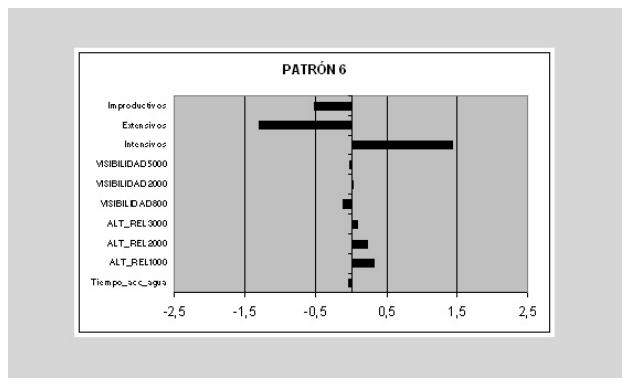

Fig 13. Media de los resultados estadísticos de los yacimientos del patrón. Los valores son relativos. 


\begin{tabular}{|l|l|}
\hline Yacimiento & Cronologia / cuituła \\
\hline Cancho Enamorado & Cogotas I-Cogotas I avanzado \\
Castillo de Carpio Bemardo & Cogetas I avanzado \\
Mesa del Carpio Bemardo & Cogotas I avanzado \\
Castil de Cabras & Hierro dudoso \\
\hline
\end{tabular}

Fig 14. Tabla con los yacimientos incluidos en el patrón.

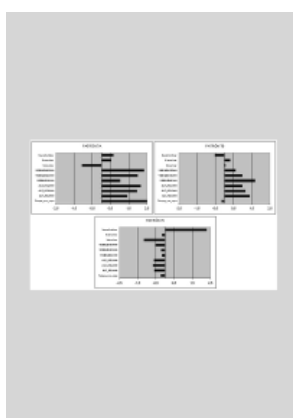

Fig 15. Media de los resultados estadísticos de los yacimientos del patrón. Los valores son relativos. 